\title{
The Regional Extent of Suppression: Strabismics Versus Nonstrabismics
}

\author{
Raiju Jacob Babu, ${ }^{1}$ Simon R. Clavagnier, ${ }^{2}$ William Bobier, ${ }^{1}$ Benjamin Thompson, ${ }^{3}$ \\ and Robert F. Hess ${ }^{2}$ \\ ${ }^{1}$ School of Optometry, University of Waterloo, Waterloo, Ontario, Canada \\ ${ }^{2}$ McGill Vision Research, Department Ophthalmology, McGill University, Montreal, Quebec, Canada \\ ${ }^{3}$ Department of Optometry and Vision Science, University of Auckland, Auckland, New Zealand
}

Correspondence: Robert F. Hess, McGill Vision Research (H4-14), Department of Ophthalmology, McGill University, PQ, Canada H3A 1A1; Robert.hess@mcgill.ca.

Submitted: November 15, 2012 Accepted: January 24, 2013

Citation: Babu RJ, Clavagnier SR, Bobier W, Thompson B, Hess RF. The regional extent of suppression: strabismics versus nonstrabismics. Invest Ophthalmol Vis Sci. 2013;54:65856593. DOI:10.1167/iovs.12-11314
Purpose. Evidence is accumulating that suppression may be the cause of amblyopia rather than a secondary consequence of mismatched retinal images. For example, treatment interventions that target suppression may lead to better binocular and monocular outcomes. Furthermore, it has recently been demonstrated that the measurement of suppression may have prognostic value for patching therapy. For these reasons, the measurement of suppression in the clinic needs to be improved beyond the methods that are currently available, which provide a binary outcome.

Methons. We describe a novel quantitative method for measuring the regional extent of suppression that is suitable for clinical use. The method involves a dichoptic perceptual matching procedure at multiple visual field locations. We compare a group of normal controls (mean age: $28 \pm 5$ years); a group with strabismic amblyopia (four with microesotropia, five with esotropia, and one with exotropia; mean age: $35 \pm 10$ years); and a group with nonstrabismic anisometropic amblyopia (mean age: $33 \pm 12$ years).

Results. The extent and magnitude of suppression was similar for observers with strabismic and nonstrabismic amblyopia. Suppression was strongest within the central field and extended throughout the $20^{\circ}$ field that we measured.

Conclusions. Suppression extends throughout the central visual field in both strabismic and anisometropic forms of amblyopia. The strongest suppression occurs within the region of the visual field corresponding to the fovea of the fixing eye.

Keywords: suppression, amblyopia, scotoma, mapping, strabismic, anisometropic

\begin{abstract}
$\mathrm{A}$ mblyopia is a clinical condition where the vision in one eye fails to develop during early childhood due to disrupted binocular function. Even though the basic binocular circuit is intact in individuals with amblyopia, strong suppression of the nonfixing eye often results in a functionally monocular visual system when both eyes are open. ${ }^{1-3}$ There is evidence to suggest that amblyopia develops as a consequence of this suppression. ${ }^{4,5}$ For example, stronger suppression is associated with deeper amblyopia at both a behavioral ${ }^{4}$ and a single cell level. ${ }^{6}$ Furthermore, there is evidence that treatment designed to reduce suppression by strengthening fusion leads to improved visual function in patients with amblyopia. ${ }^{7-10}$ Therefore, suppression may be of paramount importance in the amblyopia syndrome; however, the clinical tests currently used to assess suppression are rudimentary to say the least. ${ }^{11-13}$

Our knowledge of clinical suppression is greatest for patients with strabismic amblyopia. For these patients, suppression has traditionally been thought of as localized regions of blindness or "scotomas" affecting one eye in strategic locations such as the fovea or the region of the amblyopic field corresponding to the fovea of the fixing eye. ${ }^{11-13}$ More recently, quantitative methods have been used to measure detection thresholds in the amblyopic eye when corresponding points are stimulated in the fixing eye. ${ }^{14-17}$ The results suggest that suppression is not limited to the fovea, but encompasses a large
\end{abstract}

part of the central field $\left(10^{\circ}\right)$ with a degree of asymmetry. On the other hand, there are data which suggest that suppression can be absent in strabismic amblyopia. Mehdorn, ${ }^{18}$ using stereo perimetry, reported an absence of suppression in individuals with microstrabismus. More recently, Barrett et al., ${ }^{19}$ utilizing a blue on yellow detection task, reported an absence of suppression in the central field of strabismic amblyopes.

Our knowledge of suppression in anisometropic amblyopia, the most common nonstrabismic form of amblyopia, is less well understood. However, there is a widespread belief that suppression is weaker, or in some way different, in anisometropic relative to strabismic amblyopia. Studies into the effects of monocular optical blur in humans ${ }^{20}$ with normal vision indicate that the induced suppression extends well beyond the fovea and is related to the degree of anisometropia. This is consistent with studies of anisometropic amblyopia. For example, Sireteanu and Fronius ${ }^{17}$ reported evenly distributed suppression in human anisometropic amblyopes without any obvious asymmetry or foveal preference. In addition, Li et al. ${ }^{4}$ tested suppression within the central $22^{\circ}$ of the visual field of adults with amblyopia, and found that the degree of suppression was similar in strabismic and nonstrabismic forms of the condition. Interestingly, however, there is recent evidence that anisometropic amblyopia may be associated with weaker suppression than strabismic amblyopia in childhood. ${ }^{21}$ 


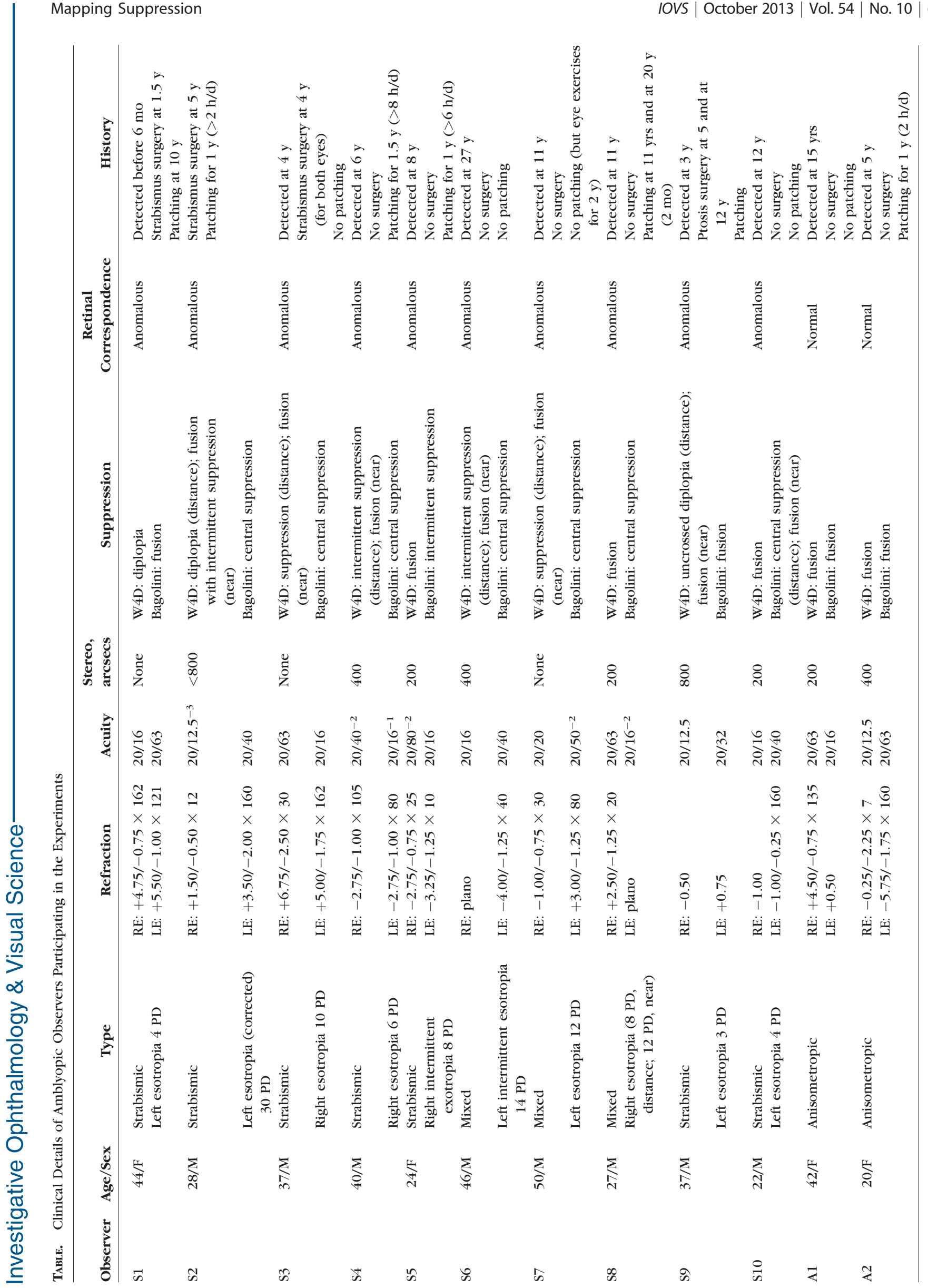


Our previous work has been directed toward developing new methods to quantify suppression ${ }^{22}$ and to compare the magnitude of suppression in strabismics and anisometropes. ${ }^{4}$ Here we direct our attention to the development of a new method for delineating the regional distribution of suppression. This method incorporates features of previous approaches in that it does not involve corresponding points, ${ }^{14-17,19}$ but provides a more global measure of suppression. ${ }^{3,22}$ The new method is novel in that it does not rely on threshold measures, ${ }^{14-17,19}$ but on suprathreshold matching. ${ }^{23}$ The technique is well suited for use in the clinic as it is relatively fast to administer and provides quantitative information on the distribution of suppression within the central visual field. We have used this new method to address two questions. First, is suppression limited to the fovea of strabismic amblyopes or does it involve all of the central $20^{\circ}$ ? Second, does the strength and regional distribution of suppression differ between strabismic and nonstrabismic forms of amblyopia?

\section{MeTHODS}

\section{Participants}

A total of 24 participants took part in this study: 10 control individuals with normal or corrected-to-normal vision (mean 28 years, SD 5 years) and 14 individuals with amblyopia (Table). Within the amblyopic group, four observers were nonstrabismic anisometropes (mean age: 33 years, SD 12 years) and 10 were strabismic amblyopes (four with microesotropia, five with esotropia, and one with exotropia; mean age: 35 years, SD 10 years). The participants were recruited from the School of Optometry Clinic at the University of Waterloo and the McGill Vision Research Unit at McGill University. The study complied with the tenets of the Declaration of Helsinki and had ethics approval from the two institutions involved. The control observers had at least 20/20 vision in each eye, normal ocular motor function, and normal stereoacuity $(<40$ arcseconds). The amblyopic participants had a difference of at least 0.2 LogMAR in visual acuity between the eyes and had impaired stereo acuity ( $>100$ arcseconds).

\section{Clinical Measurements}

Visual Acuity. Visual acuity was measured using a computerized version of the Bailey Lovie LogMAR chart (Test Chart 2000 pro and Khyber Vision, iPad application). A letterby-letter scoring procedure was adopted to obtain the visual acuity. A termination criterion of five errors on a line was used.

Stereoacuity. Stereoacuity was measured using a commercial stereoacuity test (Randot Preschool Stereo Acuity Test; Stereo Optical Co., Inc., Chicago, IL). This test provides a measurement range from 800 to 40 arcseconds.

Strabismus. Unilateral and alternate cover tests were used to determine the presence of a tropia (manifest deviation) or phoria (latent deviation) and the observed deviation, if any, was neutralized using a prism of the required magnitude (prism cover test). Amblyopic participants were classified as exotropes or esotropes based on the direction of the deviation. We did not perform visuoscopy and cannot verify the monocular fixation status of our subjects, although our dichoptic alignment procedure (described in the Regional Extent of Suppression-Dichoptic Mapping Paradigm section) provided pertinent information about their fixation centration during binocular viewing.

Worth Four-Dot Test. The test was performed at both distance $(1.6 \mathrm{~m})$ and near $(33 \mathrm{~cm})$. The test lights subtended $1^{\circ}$ of visual angle at distance and $6^{\circ}$ at near. The red and green 
filters were placed according to convention with the red filter over the right eye and the green filter over the left eye. The participants reported whether they saw all four lights and the color of each light. If the participants reported only two red or three green lights, they were considered to have complete suppression for this test. If they reported a total of five colored lights, they were considered to have diplopia; and if they reported four lights with the bottom light appearing either red or green, then they were considered to have partial suppression.

Bagolini Striated Lens Test. Suppression was qualitatively assessed using the Bagolini striated lens test. Participants viewed a point light source at two test distances $(1 \mathrm{~m}$ and $33 \mathrm{~cm}$ ) while wearing Bagolini striated lenses oriented at 45 and $135^{\circ}$ over their habitual spectacle or contact lens correction. In the instance of normal binocular vision, participants reported an " $\mathrm{X}$ " intersecting at the fixation light. In the case of complete suppression, participants only perceived one of the two lines that form an "X." In the case of a central suppression scotoma, both lines were seen but one line was incomplete.

Retinal Correspondence. The classification of retinal correspondence was based on a combination of the cover test (objective angle), the Bagolini striated lens test, and the alignment measure (subjective alignment) on the head mounted display (see Regional Extent of Suppression-Dichoptic Mapping Paradigm section). If the cover test revealed a strabismus (objective angle), but a fused central alignment was reported on the Bagolini striated lens test, then the patient was considered to have anomalous retinal correspondence. The magnitude was obtained by comparing the subjective and objective measures of the angle of deviation. If the participants reported diplopia without alignment on the Bagolini striated lens test, then they were considered to have normal retinal correspondence. We could not rule out unharmonious abnormal retinal correspondence, though it is rare.

Eye Dominance in Normal Observers. Ocular dominance was subjectively assessed using the Porta test also known as the Point-a-Finger test. Observers were asked to place the thumb of one hand over the other and extend their arms fully. Following this, they were asked to align the thumb to a 6-m target. If the target remains in view with the left eye closed, then the individual is right eye dominant. Dominance

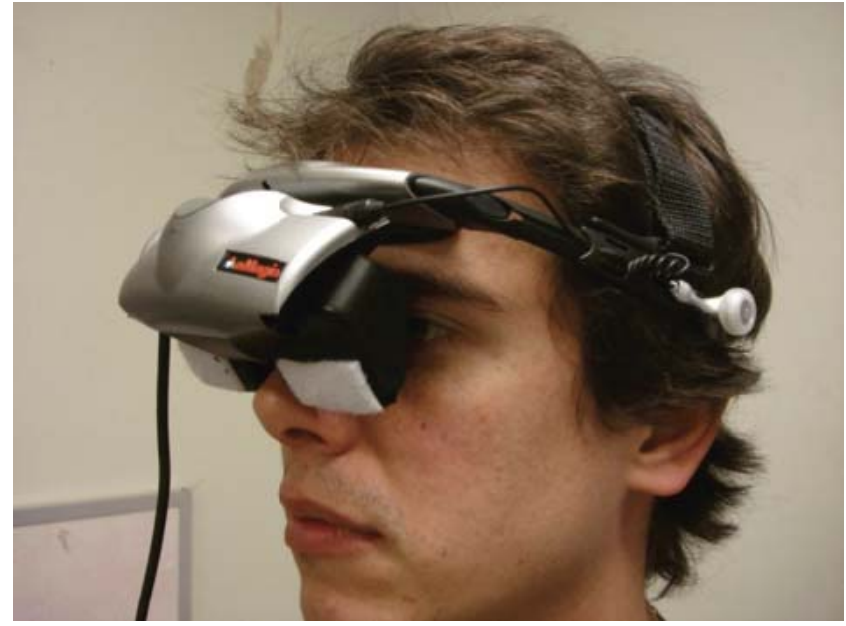

Figure 1. The head-mounted goggles used to present dichoptic stimuli for the measurement of suppression and for the restoration of binocular function. Reprinted from Hess RF, Mansouri B, Thompson B. Restoration of binocular vision in amblyopia. Strabismus. 2011;19:110-118

was determined by alternating the viewing eye to determine the sighting eye.

\section{Apparatus}

Stimuli were presented using a laptop computer (MacBook Pro; Apple Inc., Cupertino, CA) running numerical computing software (MATLAB; MathWorks Ltd., Natick, MA) and visual psychophysics software (Psychophysics Toolbox, version 3; available in the public domain at http://psychtoolbox.org). ${ }^{24,25}$ The stimuli were displayed using a dual pro head mounted display (Z800; eMagin Corp., Bellevue, WA). This head mounted display model (Fig. 1) contains two organic lightemitting diode screens that can present different stimuli to each eye. The screens have a high luminance $\left(190 \mathrm{~cd} / \mathrm{m}^{2}\right)$, a linear luminance response profile, ${ }^{22}$ and refresh simultaneously at $60 \mathrm{~Hz}$, thus avoiding motion smear. To achieve dichoptic presentation, each frame of the stimulus was computed as a single image with a resolution of $600 \times 1600$ pixels. An external video board (Matrox DuelHead2Go; Matrox Graphics
A

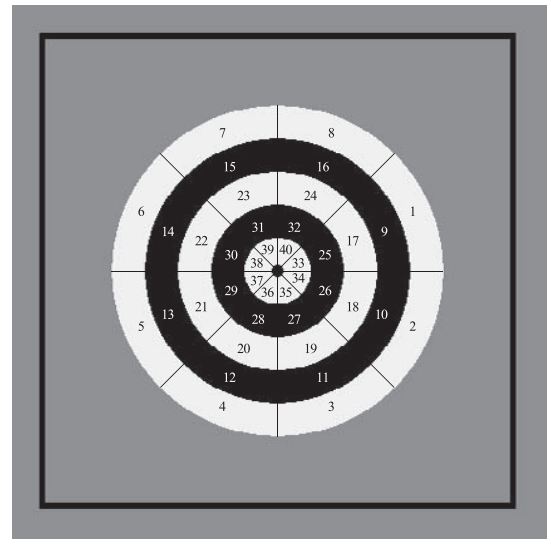

B

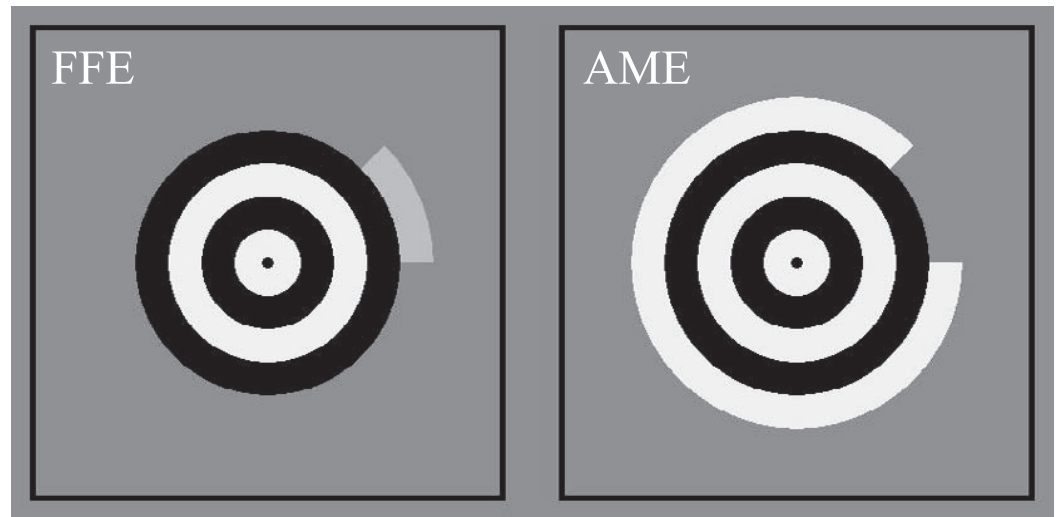

Figure 2. The suppression mapping stimulus. (A) The 40 regions of the visual field that were tested. The radius of the most eccentric ring is $10^{\circ}$. (B) Dichoptic testing arrangement. One segment was shown to the fellow fixing eye (FFE) and the remaining segments from the same annulus were shown to the amblyopic eye (AME). The observer varied the contrast of the segment shown to the fellow eye to match the perceived contrast of the segments from the same annulus shown to the amblyopic eye. The remaining annuli were shown to both eyes at $80 \%$ contrast. 

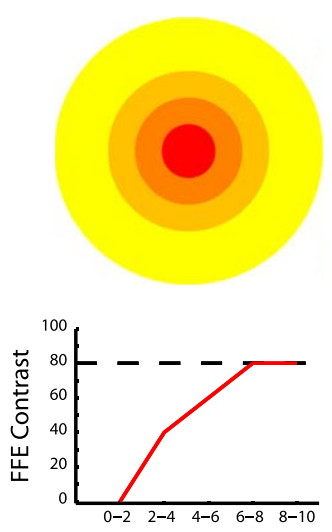

B
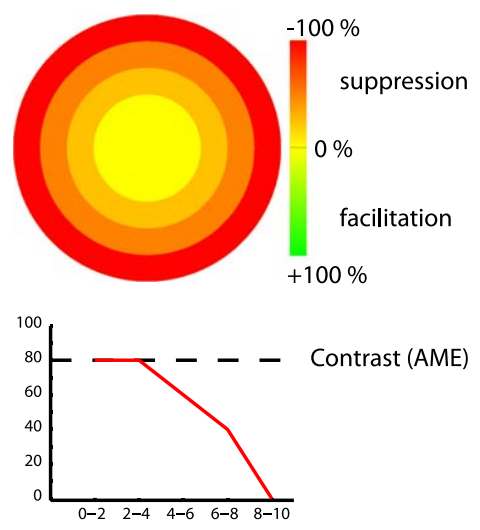

Eccentricity in deg

Figure 3. Simulated maps of pure central suppression (A) and peripheral suppression (B) within the amblyopic eye. Each point on the map corresponds to a sector in the suppression mapping stimulus shown in Figure 2A. The color bar represents the perceived difference in contrast whereby the stimulus presented to the amblyopic eye is perceived as having less (red, suppression) or more (green, facilitation) contrast than the stimulus shown to the fellow eye. The data plots below each map represent the mean matching contrast for each annulus (averaged across segments). Lower contrasts indicate stronger suppression and the slope of the line indicates whether suppression tends to be stronger centrally (positive slope) or peripherally (negative slope).
Inc., Quebec, Canada) was then used to split each frame between the two head-mounted display screens at a resolution of $600 \times 800$ pixels per screen. A photometer (United Detector Technology, Hawthorne, CA) was used to ensure equal luminance of the two screens and to perform gamma correction. ${ }^{26}$ This equipment is compact and suitable for use in the clinic. The stimuli could also be presented on a handheld device using a lenticular or anaglyph method to provide an even more compact system.

\section{Regional Extent of Suppression-Dichoptic Mapping Paradigm}

To map the suppression scotoma, we used a strategy inspired by retinotopic mapping protocols used for functional magnetic resonance imaging studies (Fig. 2). The visual stimulus $\left(20^{\circ}\right.$ diameter) was composed of five concentric annuli with alternate contrast polarities. Each annulus subtended $2^{\circ}$ of eccentricity and was divided into eight sectors, each subtending $45^{\circ}$ of visual angle. One "target" sector of variable contrast was presented to the fellow fixing eye and the seven remaining sectors on the same annulus were presented to the amblyopic eye at $80 \%$ contrast. The remaining annuli were presented to both eyes. Each dichoptic pair was aligned by the observer who used a computer keyboard to vary the position of the stimulus presented to the amblyopic eye. Once alignment was complete, the measurement of suppression began. While fixating on a central black dot, participants adjusted the contrast of the sector shown to the fellow eye until it matched
N1
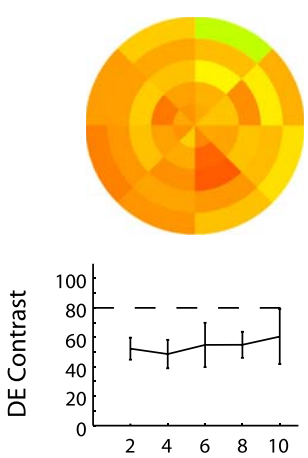

N6
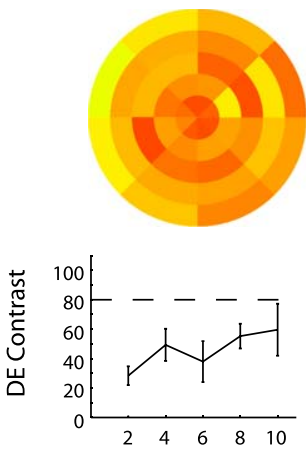

N2
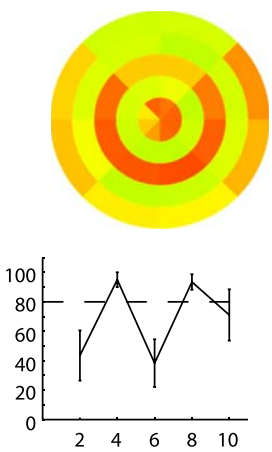

N7
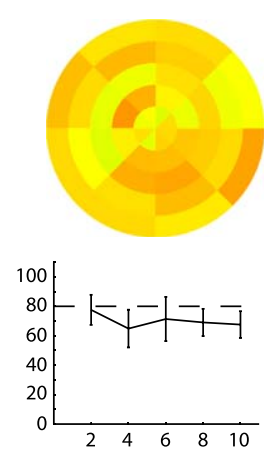

N3
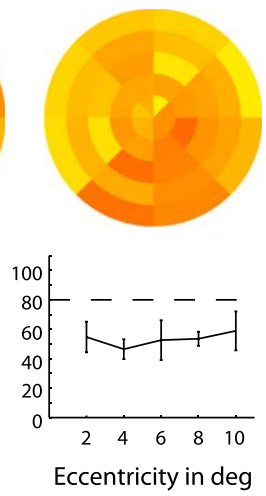

N8
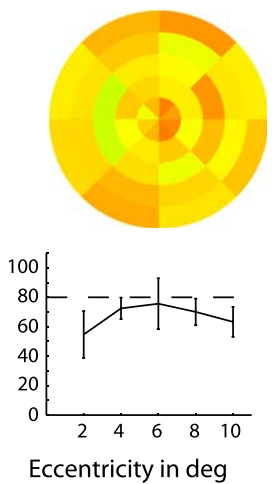

N4
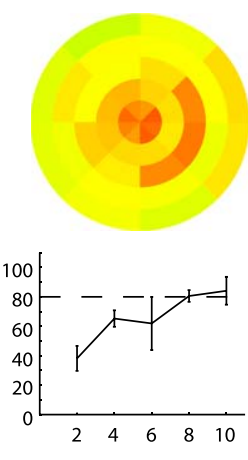

N9
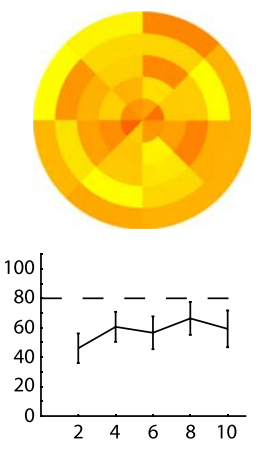

N5
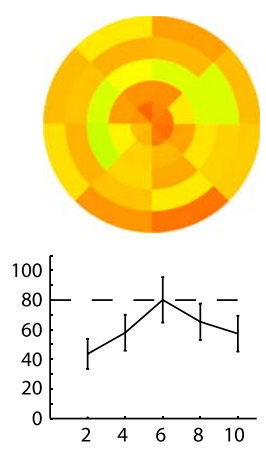

N10
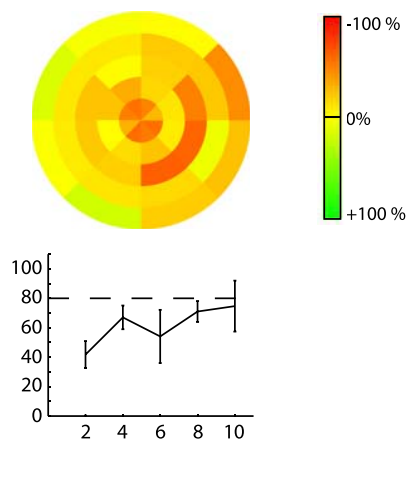

FIGURE 4. Graphical and color map representations of nondominant eye suppression across the central $20^{\circ}$ in individuals with normal binocular vision (the $x$-axis of the data plots shows the radius of the test annulus, which had a maximum value of $10^{\circ}$ relative to fixation). Data are shown for individual subjects. See Figure 3 for an explanation of the color maps. The data plots represent the mean matching contrast for each annulus (averaged across all segments). A value of $80 \%$ contrast would be expected for an exact match, as this was the contrast shown to the dominant eye (DE). Lower values indicate stronger suppression. Error bars show \pm 1 SD. 
S1
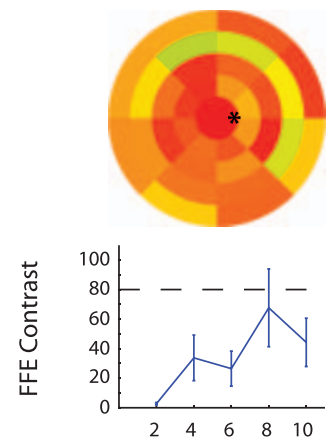

S6
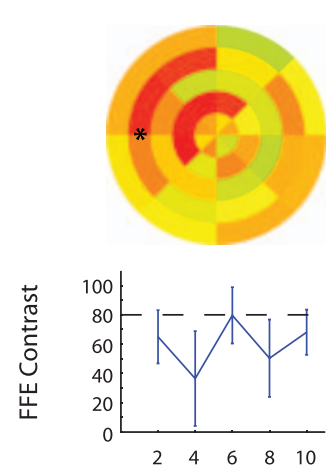

S2
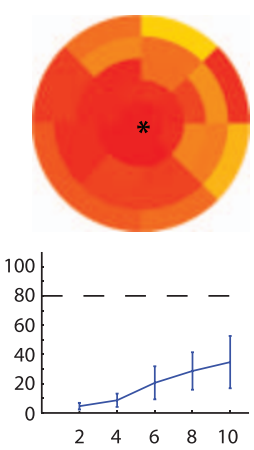

S7
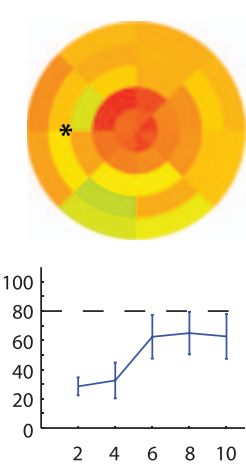

S3
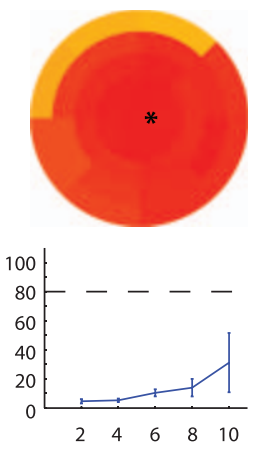

Eccentricity in deg
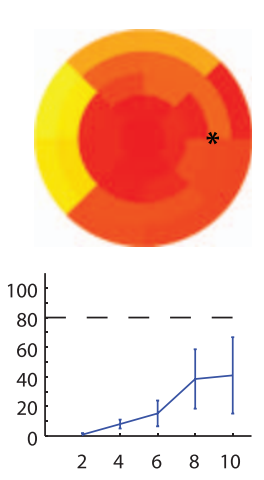

S9

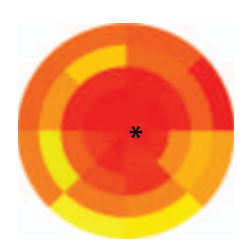

S5
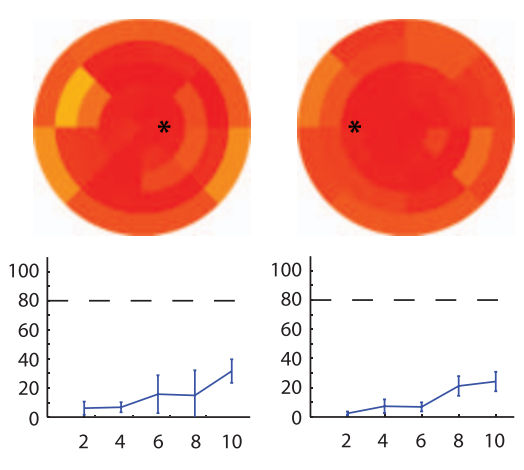

S10
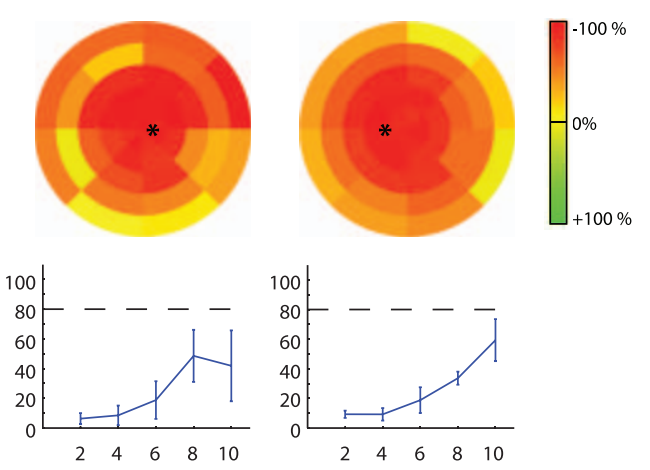

Eccentricity in deg

FIGURE 5. Suppression maps for the amblyopic eyes of 10 observers with strabismic amblyopia. See Figures 3 and 4 for details. The majority of observers showed strong suppression, particularly in the central field corresponding to the fovea of the fixing eye (center of the maps). The region marked with an asterisk represents the foveal representation in the amblyopic eye.

the perceived contrast of the remaining sectors in the annulus that were presented to the amblyopic eye. There was no time constraint. This procedure was repeated for each sector for each of the five annuli ( 40 measurements in total). The position of the sectors evolved in a predictable manner progressing from peripheral to central and following a clockwise direction on the same annulus. The matching procedure was repeated in three separate runs with the contrast step size changing from coarse to fine from one run to the next (step sizes of $10 \%, 5 \%$, and $1 \%$ contrast) to provide a final set of threshold estimates. The findings reported in the Results section represent an average of at least three complete threshold measurements per participant.

\section{Results}

To allow for visualization of the results for individual observers, the mean matching contrasts for each sector of the stimulus were plotted on a spatial map. The extent of the contrast mismatch between the two eyes, our measure of the degree of suppression, was shown using a color code with red representing contrast underestimation (suppression), green representing contrast overestimation (facilitation), and yellow representing a perfect match. Simulated results are shown in Figure 3 for pure central suppression (Fig. 3A) and pure peripheral suppression (Fig. 3B).

Dichoptic interactions similar to suppression in amblyopia are referred to as dichoptic masking for binocularly normal individuals because in normals, a stimulus in one eye can mask the detection of a different stimulus in the other eye. However, these effects in normals are typically of lesser magnitude. ${ }^{1}$ This is thought to be due to a contralateral inhibitory drive from the signals from left and right eyes prior to binocular summation. ${ }^{1}$ Maps reflecting the regional distribution of this dichoptic masking for the nondominant eyes of 10 observers with normal vision are shown in Figure 4. Below each suppression map is a plot of the mean matching contrast for each annulus that depicts the general relationship between the depth of suppression and eccentricity. While a number observers show very weak patterns of suppression and facilitation, others show regions of relatively strong suppression. This is consistent with previous studies that investigated the relationship between interocular suppression and ocular dominance, whereby a considerable number of observers with normal vision exhibited a degree of dominance. ${ }^{27,28}$

The suppression maps for the amblyopic eyes of 10 patients with strabismic amblyopia are shown in Figure 5. It is clear that the magnitude of suppression is significantly stronger in patients than in controls (Fig. 4), although there is considerable individual variability. The majority of patients show stronger suppression for the central regions of the visual field (a positive slope in the plots below each color map) although suppression is evident throughout the whole $20^{\circ}$ region for all but one patient (S6). As shown in the Table, all strabismic patients had anomalous retinal correspondence.

Results for four nonstrabismic, anisometropic amblyopes are displayed in Figure 6. A range of different suppression profiles were obtained. In two cases, the suppressed area 
A1
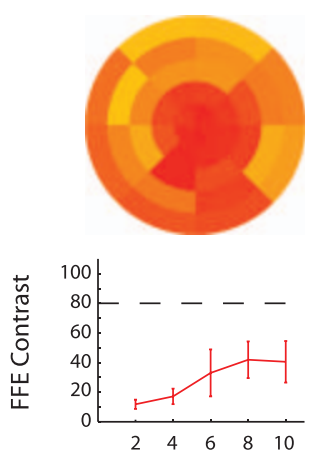

A2
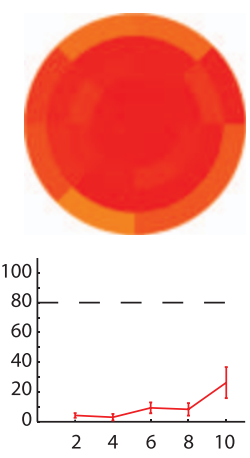

A3
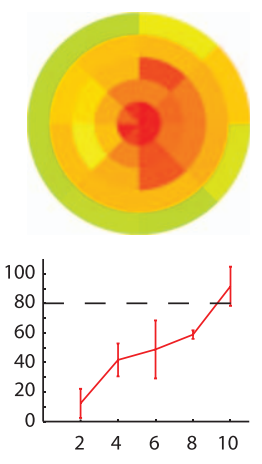

A4
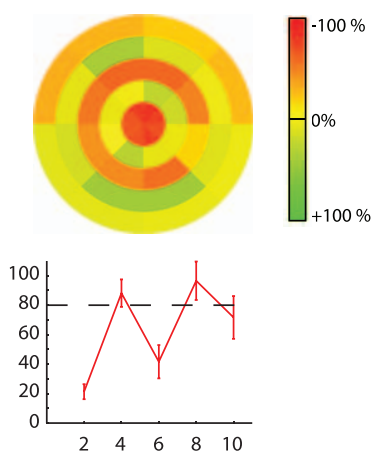

Eccentricity in deg

Figure 6. Suppression maps for four nonstrabismic anisometropic amblyopes. See Figure 3 for details.

extended across the entire testing area (A1 and A2), whereas in another two cases, it only involved the foveal representation (A3 and A4). The common feature between the strabismic (Fig. 5) and nonstrabismic (Fig. 6) amblyopes was that the central most region of the fused binocular field was always affected.

Average data for controls, strabismic amblyopes, and nonstrabismic amblyopes are shown in Figure 7. While controls showed very little suppression on average, observers with amblyopia exhibited suppression throughout the whole $20^{\circ}$ field. This suppression was particularly pronounced within the central $6^{\circ}$ of the test stimulus. A mixed ANOVA with factors of eccentricity (five levels: 0-2, 2-4, 4-6, 6-8, and 8-10) and group (strabismic amblyopes, nonstrabismic amblyopes, and controls) revealed a significant main effect of the group $\left(\mathrm{F}_{2,21}=\right.$ 13.549, $P<0.001$ ). Post hoc Bonferroni tests (corrected for multiple comparisons) revealed significant differences between controls and strabismic amblyopes $(P<0.001)$ and between controls and nonstrabismic amblyopes $(P<0.03)$. Strabismic and anisometropic amblyopes did not differ reliably from one another. There was also a significant main effect of eccentricity $\left(\mathrm{F}_{4,84}=19.46, P<0.001\right)$, whereby suppression reduced with increasing eccentricity. There was a marginal interaction between group and eccentricity $\left(\mathrm{F}_{8,84}=1.942, P=\right.$ 0.064), which was significant if the data for the strabismic and nonstrabismic amblyopes were pooled $\left(\mathrm{F}_{4}, 88=3.172, P<\right.$ 0.05). This indicates that the effect of eccentricity on the magnitude of suppression was more pronounced for amblyopes than for controls. Interestingly, we found reliable relationships between the magnitude of central suppression and the depth of amblyopia (Fig. 8A). In addition, the strength of suppression in the central region of the stimulus was correlated with the strength of suppression in the peripheral regions of the stimulus (Fig. 8B). It would appear, therefore, that the regional extent of suppression is related to the extent of monocular vision loss in the amblyopic eye.

\section{Discussion}

The aim of this study was to use a novel suprathreshold matching approach to measure the depth and regional extent
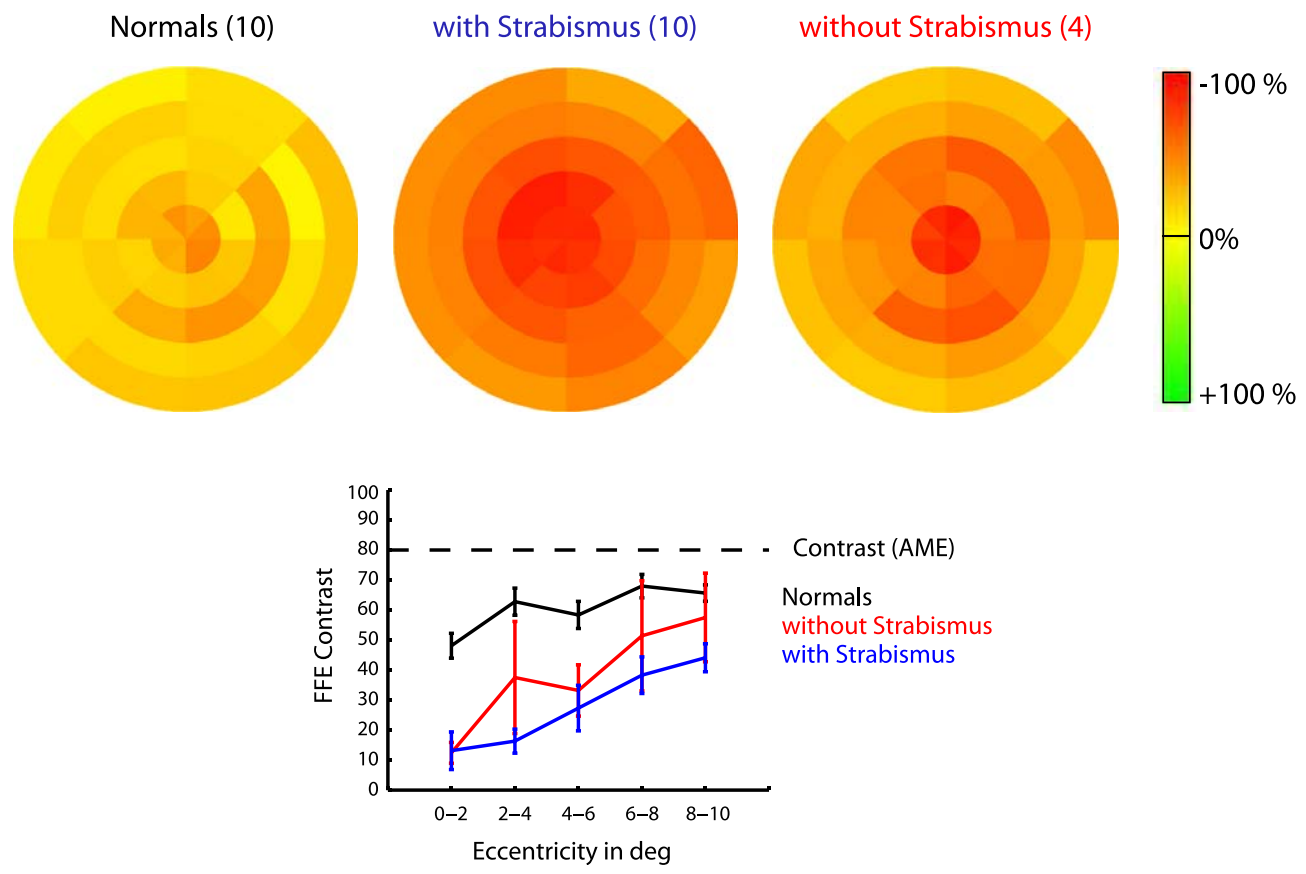

Contrast (AME)

Normals

without Strabismus

with Strabismus

FiguRE 7. Average suppression maps for observers with normal binocular vision $(n=10)$ and observers with amblyopia with $(n=10)$ and without $(n=4)$ strabismus. Observers with amblyopia had significantly stronger suppression than control observers. 
B

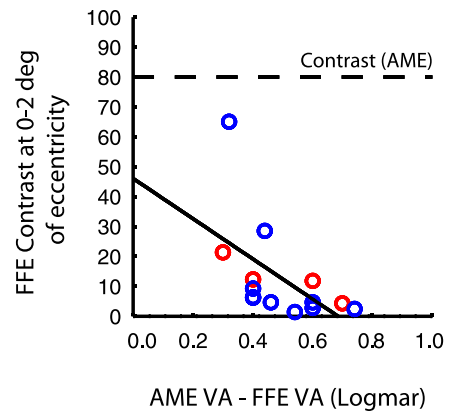

with Strabismus

without Strabismus $\quad r=-0.55$

$p<0.05$

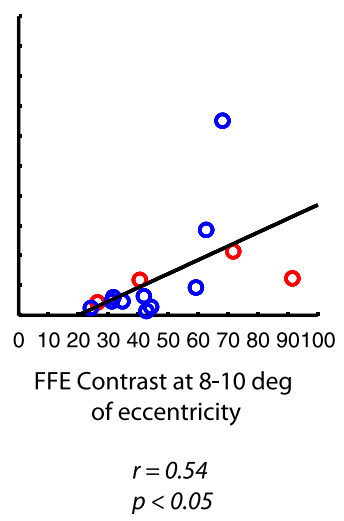

Figure 8. (A) Relationship between the magnitude of suppression (Contrast ${ }_{\mathrm{FFE}}$ ) within the central $2^{\circ}$ of the visual field and the degree of amblyopia quantified as the difference in acuity between the eyes (LogMAR AME-LogMAR FFE). (B) Relationship between the magnitude of suppression within the central $\left(0-2^{\circ}\right)$ and peripheral regions $\left(8-10^{\circ}\right)$ of the visual field. Results for the strabismic (blue symbols) and nonstrabismic (red symbols) amblyopes are displayed. Solid lines represent the best fitting linear regression and correlation coefficients with corresponding significance values are provided below each panel. VA, visual acuity.

of suppression in observers with either anisometropic or strabismic amblyopia and compare the values to a control group. Our first question related to whether suppression in strabismic amblyopia took the form of localized scotomata in regions of the amblyopic eye visual field corresponding to the foveal representation of the fixing eye. Contrary to the results of Mehdorn ${ }^{18}$ and Barrett, ${ }^{19}$ we found evidence of suppression in all the strabismic amblyopes we tested. Contrary also to the earlier results of Travers, ${ }^{13}$ Jampolosky, ${ }^{11}$ and Pratt-Johnson, ${ }^{29}$ the suppressed region was not limited to the central part of the binocular field, but was relatively diffuse, extending throughout the central $20^{\circ}$. In addition, the most pronounced suppression occurred within the region of the amblyopic eye visual field corresponding to the fovea of the fixing eye (the central most part of the field we tested). This is consistent with the measurements of Joosse and colleagues ${ }^{14-16}$ and Sireteanu and Fronius. ${ }^{5}$ Unlike Sireteanu and Fronious, ${ }^{5}$ we did not find any marked asymmetry with the nasal field being more suppressed in esotropic cases. To a first approximation, our suppression maps are symmetrical, though it is possible that had we extended our measurements to more peripheral areas, we might have seen a degree of asymmetry.

Our second question related to whether suppression is fundamentally different in the strabismic and nonstrabismic forms of amblyopia. Although we cannot provide a definitive answer to this question, we found that suppression was present in both forms of amblyopia and that the magnitude of suppression did not differ systematically between strabismic and anisometropic amblyopes. However, the variability in suppression between individuals, while not related to the type of amblyopia, was related to the visual acuity loss in the amblyopic eye. Specifically, stronger central suppression was associated with a greater acuity deficit in the amblyopic eye. These results are in agreement with an earlier report. ${ }^{4}$ The regional distribution of suppression was also similar between strabismic and nonstrabismic forms of amblyopia.

Strengths and Weaknesses. The novel suppression measurement approach we have described allows suppression to be mapped across the central $20^{\circ}$ of the visual field using a rigorous, clinically applicable psychophysical procedure. The weakness of the approach in its current form is that suppression is not measured in peripheral regions of the visual field. Accordingly, the next step in the process of assessing the role of suppression in amblyopia will be to devise a way of accurately quantifying and mapping suppression in the peripheral field. In addition, future work will include a more diverse group of strabismic observers, including constant as well as alternating exotropes and large angle esotropes.

Clinical Importance of Measuring Suppression. There is increasing evidence that suppression plays a key role in the etiology of amblyopia ${ }^{6}$ and influences the success of occlusion therapy. $^{21}$ In addition, a number of clinical studies have indicated that treatment approaches designed to reduce suppression and promote binocular fusion can significantly improve visual function in patients with amblyopia. ${ }^{7-9}$ Therefore, the quantitative measurement of suppression may provide valuable clinical information and allow for accurate monitoring of treatment efficacy. However, currently available clinical tests such as the Worth 4 dot and the Bagolini striated lenses are primarily designed to identify the presence or absence of suppression and do not provide detailed, quantitative information relating to the magnitude and regional distribution of suppression. The method we have described here provides a precise, qualitative measurement of suppression in different parts of the visual field and therefore could inform both the clinical evaluation and the treatment of patients with amblyopia.

\section{Acknowledgments}

We thank William Beaudot of Khyber Vision for his programming. Supported by CIHR Grant MOP 53346 (RFH).

Disclosure: R.J. Babu, None; S.R. Clavagnier, None; W. Bobier, None; B. Thompson, None; R.F. Hess, None

\section{References}

1. Baker DH, Meese TS, Hess RF. Contrast masking in strabismic amblyopia: attenuation, noise, interocular suppression and binocular summation. Vision Res. 2008;48:1625-1640.

2. Baker DH, Meese TS, Mansouri B, Hess RF. Binocular summation of contrast remains intact in strabismic amblyopia. Invest Ophthalmol Vis Sci. 2007;48:5332-5338.

3. Mansouri B, Thompson B, Hess RF. Measurement of suprathreshold binocular interactions in amblyopia. Vision Res. 2008; 48:2775-2784.

4. Li J, Thompson B, Lam CSY, et al. The role of suppression in amblyopia. Invest Opbthalmol Vis Sci. 2011;52:4167-4176.

5. Sireteanu R, Fronius M. Naso-temporal asymmetries in human amblyopia consequence of long-term interocular suppression. Vision Res. 1981;21:1055-1063.

6. Bi H, Zhang B, Tao X, Harwerth RS, Smith III EL, Chino YM. Neuronal responses in visual area V2 (V2) of macaque monkeys with strabismic amblyopia. Cereb Cortex. 2011;21: 2033-2045.

7. Hess RF, Mansouri B, Thompson B. A new binocular approach to the treatment of Amblyopia in adults well beyond the critical period of visual development. Restorative Neurol Neurosci. 2010;28:793-802.

8. Hess RF, Mansouri B, Thompson BA. Binocular approach to treating amblyopia: anti-suppression therapy. Optom Vis Sci. 2010;87:697-704.

9. Hess RF, Mansouri B, Thompson B. Restoration of binocular vision in amblyopia. Strabismus. 2011;19:110-118. 
10. To L, Thompson B, Blum J, Maehara G, Hess RF, Cooperstock J. A game platform for treatment of amblyopia. IEEE Trans Neural Syst Rehabil Eng. 2011;19:280-289.

11. Jampolsky A. Characteristics of suppression in strabismus. AMA Arch Ophthalmol. 1955;54:683-696.

12. Pratt-Johnson JA, Wee HS, Ellis S. Suppression associated with esotropia. Can J Ophthalmol. 1967;2:284-291.

13. Travers T. Suppression of vision in squint and its association with retinal correspondence and amblyopia. BrJ Ophthalmol. 1938;22:577-604.

14. Joosse MV, Simonsz HJ, Spekreijse H, Mulder PG, van Minderhout HM. The optimal stimulus to elicit suppression in smallangle convergent strabismus. Strabismus. 2000;8:233-242.

15. Joosse MV, Simonsz HJ, van Minderhout EM, Mulder PG, de Jong PT. Quantitative visual fields under binocular viewing conditions in primary and consecutive divergent strabismus. Graefes Arch Clin Exp Ophthalmol. 1999;237:535-545.

16. Joosse MV, Simonsz HJ, van Minderhout HM, de Jong PT, Noordzij B, Mulder PG. Quantitative perimetry under binocular viewing conditions in microstrabismus. Vision Res. 1997; 37:2801-2812.

17. Sireteanu R, Fronius M, Singer W. Binocular interaction in the peripheral visual field of humans with strabismic and anisometropic amblyopia. Vision Res. 1981;21:1065-1074.

18. Mehdorn E. Suppression scotomas in primary microstrabismus-a perimetric artefact. Doc Ophthalmol. 1989;71:1-18.

19. Barrett BT, Panesar GK, Scally AJ, Pacey IE. A limited role for suppression in the central field of individuals with strabismic amblyopia. PLoS One. 2012;7:e36611.
20. Simpson T. The suppression effect of simulated anisometropia. Ophthalmic Physiol Opt. 1991;11:350-358.

21. Narasimhan S, Harrison ER, Giaschi DE. Quantitative measurement of interocular suppression in children with amblyopia. Vision Res. 2012;66:1-10.

22. Black J, Maehara G, Thompson B, Hess RF. A compact clinical instrument for quantifying suppression. Optom Vis Sci. 2011; 88:334-342.

23. Maehara G, Thompson B, Mansouri B, Farivar R, Hess RF. The perceptual consequences of interocular suppression in amblyopia. Invest Ophthalmol Vis Sci. 52:9011-9017.

24. Brainard DH. The Psychophysics Toolbox. Spatial Vis. 1997; 10:433-436.

25. Pelli DG. The VideoToolbox software for visual psychophysics: transforming numbers into movies. Spatial Vis. 1997;10:437442.

26. Brainard DH. The Psychophysics Toolbox. Spatial Vis. 1997; 10:433-446.

27. Li J, Lam CSY, Yu M, et al. Quantifying sensory eye dominance in the normal visual system: a new technique and insights into variation across traditional tests. Invest Ophthalmol Vis Sci. 2010;51:6875-6881.

28. Zhang P, Bobier W, Thompson B, Hess RF. Binocular balance in normal vision and its modulation by mean luminance. Optom Vis Sci. 2011;88:1072-1079.

29. Pratt-Johnson J, Wee HS. Suppression associated with exotropia. Can J Ophthalmol. 1969;4:136-144. 